\title{
OPTIMAL STOCHASTIC DESIGN FOR MULTI-PARAMETER ESTIMATION PROBLEMS
}

\author{
Hamza Soganci* ${ }^{*}$, Sinan Gezici*, and Orhan Arikan* \\ *Bilkent University, Department of Electrical and Electronics Engineering, 06800, Ankara, Turkey \\ $\sharp$ TUBITAK-SAGE, Group of Electronic Systems and Flight Disciplines, 06261, Ankara, Turkey \\ $\{$ hsoganci, gezici, oarikan\}@ee.bilkent.edu.tr
}

\begin{abstract}
In this study, we consider performance improvement of an array of fixed estimators by using stochastic design techniques. The optimal design is investigated both in the absence and presence of an average power constraint. Two different performance criteria are considered; the average Bayes risk and the maximum Bayes risk. It is shown that the optimal stochastic parameter design results in a randomization between different numbers of parameter values depending on the type of the performance criterion.
\end{abstract}

Index Terms - Stochastic parameter design, Bayes risk, parameter estimation.

\section{INTRODUCTION}

In conventional estimation problems, the aim is to design the optimal estimator for a given distribution of observations in order to minimize a certain cost function. The estimators considered in these conventional formulations can be categorized into two groups based on the prior information about the parameter to be estimated. If there exists a prior information about the parameter, Bayesian estimators, such as the minimum mean-squared error (MMSE) estimator and the minimum mean-absolute error (MMAE) estimator, are commonly used [1]. On the other hand, when there is no prior information about the parameter, the minimum variance unbiased estimator (MVUE) or the maximum likelihood estimator (MLE) can be employed [2]. All these approaches require designing the optimal estimator under certain constraints. In a recent study, an alternative formulation is investigated by considering the stochastic design of a parameter when the estimator is fixed, and it is shown that the performance of the given estimator can be improved by optimal stochastic parameter design, which can involve randomization of the parameter between at most two different values [3].

Signal randomization has also been employed in various frameworks to improve the performance of detection and estimation systems [4]-[9]. For example, the detection probability of certain detectors can be increased by the addition of a randomized noise component $[4,5]$. Also, transmitting randomized signals can reduce the probability of error for power constrained communication systems in the presence of nonGaussian noise [7]. In addition, performance of some suboptimal estimators can also be enhanced by adding randomized noise to the observations before the estimation process [9].

In this study, the aim is to propose a framework for the optimal stochastic design of multiple parameters. In this way, the approach in [3] for the single parameter case is extended to the multi-parameter scenarios, in which the optimal stochastic design of multiple parameters is performed in order to optimize the performance of fixed estimators. Two different performance criteria, the total Bayes risk and the maximum Bayes risk, are considered, and the characteristics of optimal parameter distributions are specified. A numerical example is presented in order to illustrate the improvements achievable via the proposed approach.

\section{STOCHASTIC DESIGN FOR MULTIPLE PARAMETERS}

In this section, the aim is to establish a framework for the stochastic design of multiple parameters for a given set of fixed estimators. Without loss of generality, all the formulations and figures will be presented for the two parameter case for the sake of simplicity. It is possible to extend the results to the cases with more than two parameters.

Consider a parameter estimation scenario in which there exist two parameters, $\boldsymbol{\theta}_{1}$ and $\boldsymbol{\theta}_{2}$. The aim is to send information about these two parameters from two separate devices, $A_{1}$ and $A_{2}$, to two devices, $B_{1}$ and $B_{2}$, respectively, over additive noise channels as depicted in Fig. 1. Unlike a standard estimation scenario, parameter $\boldsymbol{\theta}_{i}$ is not necessarily transmitted as it is. But instead, device $A_{i}$ can transmit any function of $\boldsymbol{\theta}_{i}$, say $\mathbf{s}_{\boldsymbol{\theta}_{i}}$. Function $\mathbf{s}_{\boldsymbol{\theta}_{i}}$ can be of any type; it can be a deterministic function of $\boldsymbol{\theta}_{i}$, or it can be a stochastic function as well. The aim of this study is to find the optimal $\mathbf{s}_{\boldsymbol{\theta}_{i}}$, i.e., the optimal distribution of $\mathbf{s}_{\boldsymbol{\theta}_{i}}$, for each $\boldsymbol{\theta}_{i}$.

It should be noted that the difference between the single parameter case considered in [3] and the multi-parameter case investigated in this study is not only related to the number of parameters. The proposed multi-parameter formulation also takes into account the possible interference between the parameter related signals, as shown by the dashed cross lines in Fig. 1. Considering two parameters, the received signal (observation) at device $B_{i}$ can be expressed as

$$
\mathbf{y}_{i}=\mathbf{s}_{\boldsymbol{\theta}_{i}}+\sum_{\substack{j=1 \\ j \neq i}}^{2} \rho \mathbf{s}_{\boldsymbol{\theta}_{j}}+\mathbf{n}_{i}
$$

for $i \in\{1,2\}$, where $\rho$ is the multiplier that is set according to the interference between the parameter related signals, and $\mathbf{n}_{i}$ denotes the channel noise, which has a probability density function (PDF) represented by $p_{\mathbf{n}_{i}}(\cdot)$. Each device $B_{i}$ tries to estimate $\boldsymbol{\theta}_{i}$ based on the corresponding observation $\mathbf{y}_{i}$ specified by (1). It is assumed that these devices employ fixed estimators specified by $\hat{\boldsymbol{\theta}}_{i}\left(\mathbf{y}_{i}\right)$ in order to estimate $\boldsymbol{\theta}_{i}$. Considering parameters $\boldsymbol{\theta}_{i}$ as elements of vector $\boldsymbol{\theta}$, the prior 


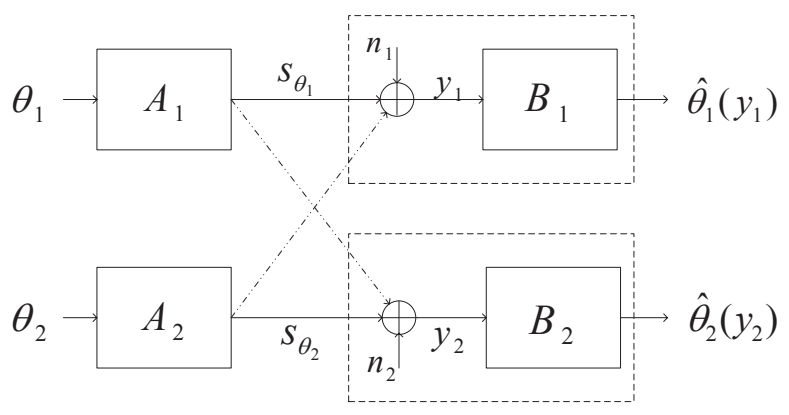

Fig. 1. System model. Devices $A_{1}$ and $A_{2}$ transmit stochastic signals $\mathbf{s}_{\boldsymbol{\theta}_{1}}$ and $\mathbf{s}_{\boldsymbol{\theta}_{2}}$ for each value of parameters $\boldsymbol{\theta}_{1}$ and $\boldsymbol{\theta}_{2}$, respectively. Devices $B_{1}$ and $B_{2}$ estimate $\boldsymbol{\theta}_{1}$ and $\boldsymbol{\theta}_{2}$ based on the noise corrupted version of $\mathbf{s}_{\boldsymbol{\theta}_{1}}$ and $\mathbf{s}_{\boldsymbol{\theta}_{2}}$.

distribution of $\boldsymbol{\theta}$ is denoted by $w(\boldsymbol{\theta})$, and the parameter space in which $\theta$ resides is represented by $\Lambda$.

The aim is to find the optimal probability distribution of $\mathbf{s}_{\boldsymbol{\theta}}$ for each $\boldsymbol{\theta} \in \Lambda$ in order to minimize a function of the Bayes risk for the given estimators, where $\mathbf{s}_{\boldsymbol{\theta}_{i}}$ 's are the elements of the vector $\mathbf{s}_{\boldsymbol{\theta}}$. Since the parameters can interfere with each other, the optimization cannot be performed independently for each parameter. Hence, the optimization should be performed jointly.

\subsection{Unconstrained Optimization}

In order to find the optimal probability distribution of $\mathbf{s}_{\boldsymbol{\theta}}$, a certain cost function is considered and the optimization is performed by minimizing that cost function. In this section, no constraints are considered for the optimization problem. In that case, the optimal stochastic parameter design can be formulated as

$$
\left\{p_{\mathbf{s}_{\boldsymbol{\theta}}}^{\mathrm{opt}}, \boldsymbol{\theta} \in \Lambda\right\}=\underset{\left\{p_{\mathbf{s}_{\boldsymbol{\theta}}}, \boldsymbol{\theta} \in \Lambda\right\}}{\arg \min } r(\hat{\boldsymbol{\theta}})
$$

where $\left\{p_{\mathbf{s}_{\boldsymbol{\theta}}}, \boldsymbol{\theta} \in \Lambda\right\}$ denotes the set of PDFs for $\mathbf{s}_{\boldsymbol{\theta}}$ for all possible values of parameter $\boldsymbol{\theta}$, and $r(\hat{\boldsymbol{\theta}})$ is the cost function for the overall system. For the single parameter case, the Bayes risk of the estimator was a natural choice for this cost function [3]. On the other hand, it is possible to consider different risk functions for the multi-parameter case. In this section, two different cost functions are considered. The first one is the sum of the Bayes risks of the estimators in the system (called the total Bayes risk), and the second one is the maximum of the Bayes risks of the estimators (called the maximum Bayes risk). For both of these cost functions, the Bayes risk of each estimator should be calculated first. For the two parameter case, the Bayes risk of the first estimator can be expressed as

$$
\begin{aligned}
& r\left(\hat{\boldsymbol{\theta}}_{1}\right)=\int_{\Lambda_{1}} w\left(\boldsymbol{\theta}_{1}\right) \int p_{\mathbf{s}_{\boldsymbol{\theta}_{1}}}\left(\mathbf{x}_{1}\right) \int \mathrm{C}\left[\hat{\boldsymbol{\theta}}\left(\mathbf{y}_{1}\right), \boldsymbol{\theta}_{1}\right] \\
& \times \int p_{\mathbf{s}_{\boldsymbol{\theta}_{2}}}\left(\mathbf{x}_{2}\right) p_{\mathbf{n}_{1}}\left(\mathbf{y}_{1}-\mathbf{x}_{1}-\rho \mathbf{x}_{2}\right) d \mathbf{x}_{2} d \mathbf{y}_{1} d \mathbf{x}_{1} d \boldsymbol{\theta}_{1}
\end{aligned}
$$

where $\mathrm{C}\left[\hat{\boldsymbol{\theta}}\left(\mathbf{y}_{1}\right), \boldsymbol{\theta}_{1}\right]$ represents the cost of estimating $\boldsymbol{\theta}_{1}$ as $\hat{\boldsymbol{\theta}}\left(\mathbf{y}_{1}\right)$ [2], and $p_{\mathbf{s}_{\boldsymbol{\theta}_{i}}}$ is the PDF of the signal related to parameter $i$. (The Bayes risk of the second estimator can be expressed similarly.) Defining an auxiliary function $g_{\boldsymbol{\theta}_{1}}(\mathbf{x})$ for the first estimator as

$$
g_{\boldsymbol{\theta}_{1}}(\mathbf{x}) \triangleq \int \mathrm{C}\left[\hat{\boldsymbol{\theta}}_{1}\left(\mathbf{y}_{1}\right), \boldsymbol{\theta}_{1}\right] p_{\mathbf{n}_{1}}\left(\mathbf{y}_{1}-\mathbf{x}_{1}-\rho \mathbf{x}_{2}\right) d \mathbf{y}_{1}
$$

where $\mathbf{x}=\left[\begin{array}{ll}\mathbf{x}_{1} & \mathbf{x}_{2}\end{array}\right]$, and a similar function for the second estimator, the total Bayes risk can be stated as

$$
\begin{aligned}
r(\hat{\boldsymbol{\theta}}) & =\int_{\Lambda} w(\boldsymbol{\theta}) \int p_{\mathbf{s}_{\boldsymbol{\theta}}}(\mathbf{x})\left(g_{\boldsymbol{\theta}_{1}}(\mathbf{x})+g_{\boldsymbol{\theta}_{2}}(\mathbf{x})\right) d \mathbf{x} d \boldsymbol{\theta} \\
& =\int_{\Lambda} w(\boldsymbol{\theta}) \mathrm{E}\left\{\tilde{g_{\boldsymbol{\theta}}}\left(\mathbf{s}_{\boldsymbol{\theta}}\right)\right\} d \boldsymbol{\theta}
\end{aligned}
$$

where $\hat{\boldsymbol{\theta}}=\left[\hat{\boldsymbol{\theta}}_{1} \hat{\boldsymbol{\theta}}_{2}\right]$ and

$$
\tilde{g}_{\boldsymbol{\theta}}(\mathbf{x})=g_{\boldsymbol{\theta}_{1}}(\mathbf{x})+g_{\boldsymbol{\theta}_{2}}(\mathbf{x}) .
$$

In (5), each expectation operation is over the PDF of $\mathbf{s}_{\boldsymbol{\theta}}$ for a given value of $\boldsymbol{\theta}$. When there are no constraints on the design of $\mathbf{s}_{\boldsymbol{\theta}}, r(\hat{\boldsymbol{\theta}})$ given by (5) can be minimized if the PDF of $\mathbf{s}_{\boldsymbol{\theta}}$ assigns all the probability to the minimizer of $\tilde{g}_{\boldsymbol{\theta}}$ in (6) for each $\boldsymbol{\theta}{ }^{1}$ In other words, the solution of the optimization problem in (2) can be simplified as

$$
p_{\mathbf{s}_{\boldsymbol{\theta}}}^{\mathrm{opt}}(\mathbf{x})=\delta\left(\mathbf{x}-\mathbf{s}_{\boldsymbol{\theta}}^{\mathrm{unc}}\right), \mathbf{s}_{\boldsymbol{\theta}}^{\mathrm{unc}}=\arg \min _{\mathbf{x}} \tilde{g}_{\boldsymbol{\theta}}(\mathbf{x})
$$

for all $\boldsymbol{\theta} \in \Lambda$. Since the solution requires $\mathbf{s}_{\boldsymbol{\theta}}$ to assign all the probability to a single point, it can be concluded that optimal PDFs for the stochastic parameter design are the ones with single point masses. Hence, the deterministic parameter design is optimal and there is no need for stochastic modeling. Also it can be observed from (7) that the solution is independent of the prior distribution $w(\boldsymbol{\theta})$, since the optimal solution is obtained for each $\boldsymbol{\theta}$ separately.

When the maximum Bayes risk criterion is considered, the cost function in (5) can be updated as

$$
\begin{aligned}
r(\hat{\boldsymbol{\theta}}) & =\int_{\Lambda} w(\boldsymbol{\theta}) \max _{i \in\{1,2\}}\left(\int p_{\mathbf{s}_{\boldsymbol{\theta}}}(\mathbf{x}) g_{\boldsymbol{\theta}_{i}}(\mathbf{x}) d \mathbf{x}\right) d \boldsymbol{\theta} \\
& =\int_{\Lambda} w(\boldsymbol{\theta}) \max _{i \in\{1,2\}}\left(\mathrm{E}\left\{g_{\boldsymbol{\theta}_{i}}\left(\mathbf{s}_{\boldsymbol{\theta}}\right)\right\}\right) d \boldsymbol{\theta}
\end{aligned}
$$

for which the solution is still a PDF with a single point mass. Hence, for the unconstrained case, considering the total risk or the maximum risk does not change the structure of the solution. However, in practice, the values of $\mathbf{s}_{\boldsymbol{\theta}}$ cannot be chosen without any constraints. In the next section, the cases with average power constraints are investigated.

\subsection{Constrained Optimization}

In this section, an average power constraint is considered in the formulation of the stochastic design problem. Although this is a specific type of a constraint, other types of constraints can also be incorporated into the theoretical analysis as well.

Consider an average power constraint in the form of

$$
\mathrm{E}\left\{\left\|\mathbf{s}_{\boldsymbol{\theta}}\right\|^{2}\right\} \leq A_{\boldsymbol{\theta}}
$$

for $\boldsymbol{\theta} \in \Lambda$, where $\left\|\mathbf{s}_{\boldsymbol{\theta}}\right\|$ is the Euclidean norm of vector $\mathbf{s}_{\boldsymbol{\theta}}$, and $A_{\boldsymbol{\theta}}$ denotes the average power constraint for $\boldsymbol{\theta}$. In general, constraint $A_{\boldsymbol{\theta}}$ can be a function of $\boldsymbol{\theta}$.

\footnotetext{
${ }^{1}$ If there are multiple minimizers, any (combination) of them can be chosen for the optimal solution.
} 
From (5) and (9), the optimal stochastic parameter design problem for the total Bayes risk criterion can be stated as

$$
\begin{gathered}
\min _{\left\{p_{\mathbf{s}_{\boldsymbol{\theta}}}, \boldsymbol{\theta} \in \Lambda\right\}} \int_{\Lambda} w(\boldsymbol{\theta}) \mathrm{E}\left\{\tilde{g}_{\boldsymbol{\theta}}\left(\mathbf{s}_{\boldsymbol{\theta}}\right)\right\} d \boldsymbol{\theta} \\
\text { subject to } \mathrm{E}\left\{\left\|\mathbf{s}_{\boldsymbol{\theta}}\right\|^{2}\right\} \leq A_{\boldsymbol{\theta}}, \forall \boldsymbol{\theta} \in \Lambda
\end{gathered}
$$

where $\tilde{g}_{\boldsymbol{\theta}}(\cdot)$ is as defined in (6). Due to the structure of the objective function and the constraint, the constrained optimization problem in (10) can be solved separately for each $\boldsymbol{\theta}$ as

$$
\min _{p_{\mathbf{s}_{\boldsymbol{\theta}}}} \mathrm{E}\left\{\tilde{g_{\boldsymbol{\theta}}}\left(\mathbf{s}_{\boldsymbol{\theta}}\right)\right\} \text { subject to } \mathrm{E}\left\{\left\|\mathbf{s}_{\boldsymbol{\theta}}\right\|^{2}\right\} \leq A_{\boldsymbol{\theta}}
$$

for $\boldsymbol{\theta} \in \Lambda$. Hence, the solution does not depend on the prior distribution $w(\boldsymbol{\theta})$.

When the maximum Bayes risk criterion is considered, the problem in (11) takes the following form:

$$
\min _{p_{\mathbf{s}_{\boldsymbol{\theta}}}} \max _{i \in\{1,2\}}\left(\mathrm{E}\left\{g_{\boldsymbol{\theta}_{i}}\left(\mathbf{s}_{\boldsymbol{\theta}}\right)\right\}\right) \text { subject to } \mathrm{E}\left\{\left\|\mathbf{s}_{\boldsymbol{\theta}}\right\|^{2}\right\} \leq A_{\boldsymbol{\theta}}
$$

Similar optimization problems in the form of (11) and (12) have been investigated in the literature [3]-[4]. The problem in (11) has the same form as the one considered in [3]. Therefore, the statistical behavior of the optimal solution is the same; that is, the optimal solution can be achieved by a randomization between at most two different values of $\boldsymbol{\theta}$. Then, the optimal solution can be obtained based on a similar approach to that in [3]. On the other hand, the problem in (12) has a different form than that in [3]. Based on arguments similar to those in [11], the following result can be obtained.

Proposition 1: Suppose $g_{\theta}$ is a continuous function and each component of $\mathbf{s}_{\boldsymbol{\theta}}$ resides in a finite closed interval. Then, an optimal solution to (12) can be expressed as

$$
p_{\mathbf{s}_{\boldsymbol{\theta}}}^{\mathrm{opt}}(\mathbf{x})=\sum_{j=1}^{3} \lambda_{\boldsymbol{\theta}_{j}} \delta\left(\mathbf{x}-\mathbf{s}_{\boldsymbol{\theta}_{j}}\right)
$$

where $\lambda_{\boldsymbol{\theta}_{j}} \geq 0$ and $\sum_{j=1}^{3} \lambda_{\boldsymbol{\theta}_{j}}=1$.

Proof: Consider the set of all $\left(g_{\boldsymbol{\theta}_{1}}\left(\mathbf{s}_{\boldsymbol{\theta}}\right), g_{\boldsymbol{\theta}_{2}}\left(\mathbf{s}_{\boldsymbol{\theta}}\right),\left\|\mathbf{s}_{\boldsymbol{\theta}}\right\|^{2}\right)$ triplets and the set of all $\left(\mathrm{E}\left\{g_{\boldsymbol{\theta}_{1}}\left(\mathbf{s}_{\boldsymbol{\theta}}\right)\right\}, \mathrm{E}\left\{g_{\boldsymbol{\theta}_{2}}\left(\mathbf{s}_{\boldsymbol{\theta}}\right)\right\}, \mathrm{E}\left\{\left\|\mathbf{s}_{\boldsymbol{\theta}}\right\|^{2}\right\}\right)$ triplets, and denote them as $U$ and $W$, respectively. Namely, $U=\left\{\left(u_{1}, u_{2}, u_{3}\right): u_{1}=g_{\boldsymbol{\theta}_{1}}\left(\mathbf{s}_{\boldsymbol{\theta}}\right), u_{2}=g_{\boldsymbol{\theta}_{2}}\left(\mathbf{s}_{\boldsymbol{\theta}}\right), u_{3}=\right.$ $\left.\left\|\mathbf{s}_{\boldsymbol{\theta}}\right\|^{2}, \forall \mathbf{s}_{\boldsymbol{\theta}}\right\}$ and $W=\left\{\left(w_{1}, w_{2}, w_{3}\right): w_{1}=\mathrm{E}\left\{g_{\boldsymbol{\theta}_{1}}\left(\mathbf{s}_{\boldsymbol{\theta}}\right)\right\}\right.$, $\left.w_{2}=\mathrm{E}\left\{g_{\boldsymbol{\theta}_{2}}\left(\mathbf{s}_{\boldsymbol{\theta}}\right)\right\}, w_{3}=\mathrm{E}\left\{\left\|\mathbf{s}_{\boldsymbol{\theta}}\right\|^{2}\right\}, \forall p_{\mathbf{s}_{\boldsymbol{\theta}}}\right\}$. As in [4], [7], and [11], it can be shown that the convex hull of $U$ is equal to $W$. Then, based on Carathéodory's theorem [12], it is concluded that any point in $W$ can be expressed as a convex combination of at most four points in $U$. In addition, since an optimal PDF should achieve the minimum value, it must correspond to the boundary of $W$, resulting in a convex combination of at most three points in $U$. Therefore, an optimal solution can be expressed as in (13).

Proposition 1 states that the optimal solution can be achieved by a randomization between at most three different values for each $\boldsymbol{\theta}$. Based on this result, the optimal stochastic parameter design problem can be expressed as

$\min _{\left\{\lambda_{\boldsymbol{\theta}_{j}}, \mathbf{s}_{\boldsymbol{\theta}_{j}}\right\}_{j=1}^{3}} \max _{i \in\{1,2\}}\left(\sum_{j=1}^{3} \lambda_{\boldsymbol{\theta}_{j}} g_{\boldsymbol{\theta}_{i}}\left(\mathbf{s}_{\boldsymbol{\theta}_{j}}\right)\right)$

subject to $\sum_{j=1}^{3} \lambda_{\boldsymbol{\theta}_{j}}\left\|\mathbf{s}_{\boldsymbol{\theta}_{j}}\right\|^{2} \leq A_{\boldsymbol{\theta}}, \sum_{j=1}^{3} \lambda_{\boldsymbol{\theta}_{j}}=1, \lambda_{\boldsymbol{\theta}_{j}} \in[0,1]$ for $\boldsymbol{\theta} \in \Lambda$. Compared to (12), the formulation in (14) provides a significant simplification as it requires optimization over a finite number of variables instead of over all possible PDFs. Since generic cost functions and noise distributions are considered in the theoretical analysis, $g_{\boldsymbol{\theta}_{1}}$ and $g_{\boldsymbol{\theta}_{2}}$ in (4) are quite generic and the optimization problem in (14) can be nonconvex in general. Therefore, global optimization techniques such as particle swarm optimization (PSO) or differential evolution can be used to obtain the solution $[13,14]$.

Remark 1: In the generic multi-parameter case with $K$ parameters, the previous results can be extended as follows: For the total Bayes risk criterion, the optimal solution is in the form a probability distribution with two point masses for any value of $K$. On the other hand, for the maximum Bayes risk criterion, the optimal solution is achieved by randomization among at most $(K+1)$ different values of $\boldsymbol{\theta}$.

\section{NUMERICAL RESULTS}

For a numerical example, consider an estimation problem in which parameter vector $\boldsymbol{\theta}=\left[\boldsymbol{\theta}_{1} \boldsymbol{\theta}_{2}\right]^{T}$ is to be estimated based on observation vector $\mathbf{y}=\left[\begin{array}{ll}\mathbf{y}_{1} & \mathbf{y}_{2}\end{array}\right]^{T}$, which is modeled as

$$
\mathbf{y}=\mathbf{s}_{\boldsymbol{\theta}}+\rho(\mathbf{1}-\mathbf{I}) \mathbf{s}_{\boldsymbol{\theta}}+\mathbf{n}
$$

where $\mathbf{s}_{\boldsymbol{\theta}}=\left[\begin{array}{ll}\mathbf{s}_{\boldsymbol{\theta}_{1}} & \mathbf{s}_{\boldsymbol{\theta}_{2}}\end{array}\right]^{T}, \mathbf{n}=\left[\mathbf{n}_{1} \mathbf{n}_{2}\right]^{T}, \mathbf{I}$ is the identity matrix of size $2 \times 2$, and $\mathbf{1}$ is the matrix of ones with the same size. $\mathbf{n}$ is the additive noise component, where $\mathbf{n}_{1}$ and $\mathbf{n}_{2}$ are independent and identically distributed Gaussian random variables, specified by PDFs $p_{\mathbf{n}_{1}}(\mathbf{n})=p_{\mathbf{n}_{2}}(\mathbf{n})=\exp \{-(\mathbf{n}-$ $\left.\mu)^{2} /\left(2 \sigma^{2}\right)\right\} /(\sqrt{2 \pi} \sigma)$. The estimator is given by $\hat{\boldsymbol{\theta}}(\mathbf{y})=\mathbf{y}$ where each parameter is estimated independently based on the corresponding observation. The cost function for each parameter is selected as the uniform cost function, which is expressed as $\mathrm{C}\left[\hat{\boldsymbol{\theta}}_{i}\left(\mathbf{y}_{i}\right), \boldsymbol{\theta}_{i}\right]=1$ if $\left|\hat{\boldsymbol{\theta}}_{i}\left(\mathbf{y}_{i}\right)-\boldsymbol{\theta}_{i}\right|>\Delta$ and $\mathrm{C}\left[\hat{\boldsymbol{\theta}}_{i}\left(\mathbf{y}_{i}\right), \boldsymbol{\theta}_{i}\right]=0$ otherwise for $i=1,2$. Based on this model, $g_{\boldsymbol{\theta}_{1}}$ in (4) can be obtained as

$$
\begin{aligned}
g_{\boldsymbol{\theta}_{1}}(\mathbf{x}) & =Q\left(\frac{\mathbf{x}_{1}+\rho \mathbf{x}_{2}-\boldsymbol{\theta}_{1}+\mu+\Delta}{\sigma}\right) \\
& +Q\left(\frac{-\mathbf{x}_{1}-\rho \mathbf{x}_{2}+\boldsymbol{\theta}_{1}-\mu+\Delta}{\sigma}\right)
\end{aligned}
$$

where $Q(\mathrm{x})=(1 / \sqrt{2 \pi}) \int_{x}^{\infty} \exp \left\{-t^{2} / 2\right\} d t$ denotes the $Q$ function. $\mathrm{E}\left\{\left\|\mathbf{s}_{\boldsymbol{\theta}}\right\|^{2}\right\} \leq\|\boldsymbol{\theta}\|^{2}$ is considered for each $\boldsymbol{\theta}$ as the constraint stated in (9). $g_{\boldsymbol{\theta}_{2}}$ for the second parameter can be obtained similarly.

For the numerical examples, the parameter spaces for both parameters are specified as $\Lambda_{1}=\Lambda_{2}=[-10,10]$. Also, $\mathrm{s}_{\theta_{i}}$ can take values in the interval $[-10,10]$ under the average power constraint, $\mathrm{E}\left\{\left\|\mathbf{s}_{\boldsymbol{\theta}_{i}}\right\|^{2}\right\} \leq\left\|\boldsymbol{\theta}_{i}\right\|^{2}$ for $i=1,2$. In addition, the Gaussian noise is taken to be zero mean with $\sigma=0.5$ and $\rho$ is chosen to be 0.25 . Since the noise is a zero-mean random variable, $\hat{\boldsymbol{\theta}}(\mathbf{y})=\mathbf{y}$ can be regarded as a practical estimator. $^{2}$ Finally, $\Delta=1$ is considered for the uniform cost function described in the previous paragraph.

\footnotetext{
${ }^{2}$ Although this is not an optimal estimator, it can be used in practice due to its simplicity compared to the optimal estimator.
} 


\begin{tabular}{|c|c|c|c|c|c|c|c|c|c|c|c|}
\hline$\theta_{1}$ & $\theta_{2}$ & $\lambda_{\boldsymbol{\theta}_{1}}$ & $\mathbf{s}_{\boldsymbol{\theta}_{1}}$ & $\lambda_{\boldsymbol{\theta}_{2}}$ & $\mathbf{s}_{\boldsymbol{\theta}_{2}}$ & $\lambda_{\boldsymbol{\theta}_{1}}$ & $\mathbf{s}_{\boldsymbol{\theta}_{1}}$ & $\lambda_{\boldsymbol{\theta}_{2}}$ & $\mathbf{s}_{\boldsymbol{\theta}_{2}}$ & $\lambda_{\boldsymbol{\theta}_{3}}$ & $\mathbf{s}_{\boldsymbol{\theta}_{3}}$ \\
\hline-5 & -5 & 1 & $(-4,-4)$ & & & 1 & $(-4,-4)$ & - & & & \\
\hline-5 & 2 & 0.721 & $(-5.42,3.09)$ & 0.279 & $(0.383,1.788)$ & 0.654 & $(-5.403,2.977)$ & 0.183 & $(0.645,1.769)$ & 0.163 & $(-4.482,-1.202)$ \\
\hline-5 & 5 & 0.513 & $(-4.525,-1.136)$ & 0.487 & $(-6.290,6.340)$ & 0.488 & $(-6.282,6.255)$ & 0.269 & $(0.771,4.767)$ & 0.243 & $(-4.560,-1.180)$ \\
\hline 5 & -2 & 0.714 & $(5.438,-3.119)$ & 0.286 & $(-0.503,-1.748)$ & 0.716 & $(5.323,-2.817)$ & 0.188 & $(-0.574,-1.743)$ & 0.116 & $(4.533,0.887)$ \\
\hline 5 & 5 & 1 & $(4,4)$ & - & - & 1 & $(4,4)$ & - & - & - & - \\
\hline
\end{tabular}

Table 1. Optimal stochastic solution $p_{\mathbf{s}_{\theta}}^{\text {opt }}(\mathbf{x})=\lambda_{\boldsymbol{\theta}_{1}} \delta\left(\mathbf{x}-\mathbf{s}_{\boldsymbol{\theta}_{1}}\right)+\lambda_{\boldsymbol{\theta}_{2}} \delta\left(\mathbf{x}-\mathbf{s}_{\boldsymbol{\theta}_{2}}\right)$ for total Bayes risk criterion (left), and optimal stochastic solution $p_{\mathbf{s}_{\boldsymbol{\theta}}}^{\mathrm{opt}}(\mathbf{x})=\lambda_{\boldsymbol{\theta}_{1}} \delta\left(\mathbf{x}-\mathbf{s}_{\boldsymbol{\theta}_{1}}\right)+\lambda_{\boldsymbol{\theta}_{2}} \delta\left(\mathbf{x}-\mathbf{s}_{\boldsymbol{\theta}_{2}}\right)+\lambda_{\boldsymbol{\theta}_{3}} \delta\left(\mathbf{x}-\mathbf{s}_{\boldsymbol{\theta}_{3}}\right)$ for the maximum Bayes risk criterion (right).

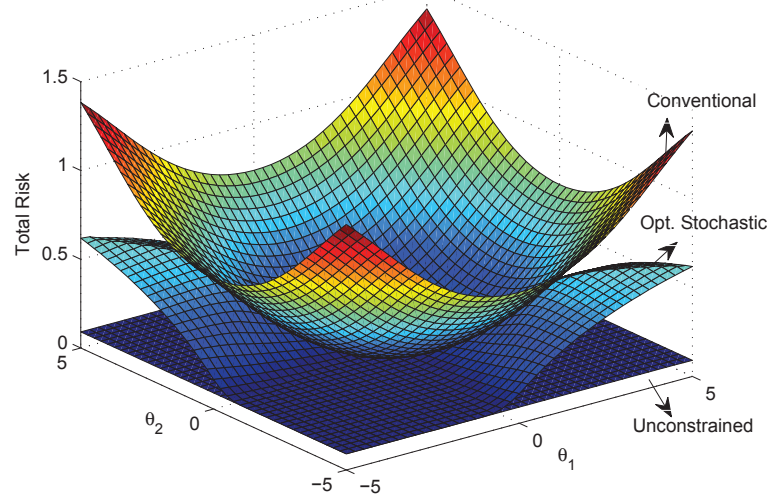

Fig. 2. Total Bayes risk versus $\boldsymbol{\theta}_{1}$ and $\boldsymbol{\theta}_{2}$.

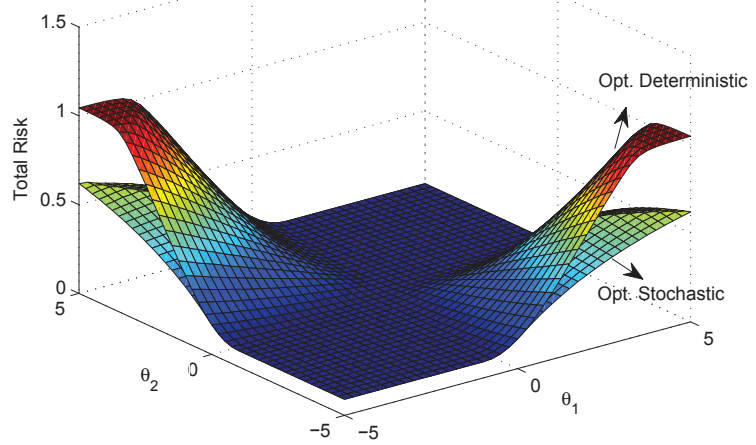

Fig. 3. Total Bayes risk versus $\boldsymbol{\theta}_{1}$ and $\boldsymbol{\theta}_{2}$.

In Fig. 2, the total Bayes risks for the stochastic parameter design, the unconstrained parameter design and the conventional parameter design (which transmits the parameters as they are; i.e., employs $\mathbf{s}_{\boldsymbol{\theta}_{i}}=\boldsymbol{\theta}_{i}$ ) are illustrated. Also in Fig. 3, the total Bayes risks for the stochastic parameter design and the deterministic parameter design (which employs no randomization) are compared. It is observed that the stochastic design achieves an improvement over the deterministic and conventional designs. Also, for some values of $\boldsymbol{\theta}_{1}$ and $\boldsymbol{\theta}_{2}$, the performance of the stochastic design is the same as the unconstrained design.

In Fig. 4, the maximum Bayes risks for the stochastic parameter design, the unconstrained parameter design and the conventional parameter design are shown. Also, in Fig. 5, the maximum Bayes risks for stochastic parameter design and the deterministic parameter design are compared. Again, similar observations as in the previous scenario are made.

In Table 1, the optimal solutions for the stochastic, the deterministic and the unconstrained parameter design ap-

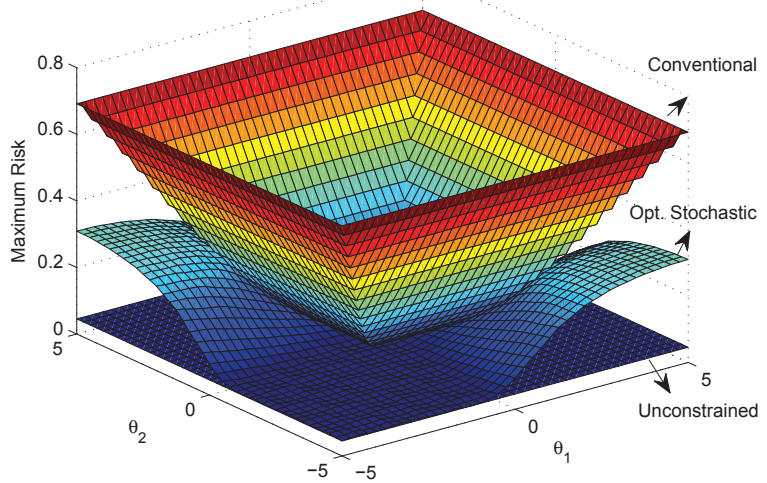

Fig. 4. Maximum Bayes risk versus $\boldsymbol{\theta}_{1}$ and $\boldsymbol{\theta}_{2}$.

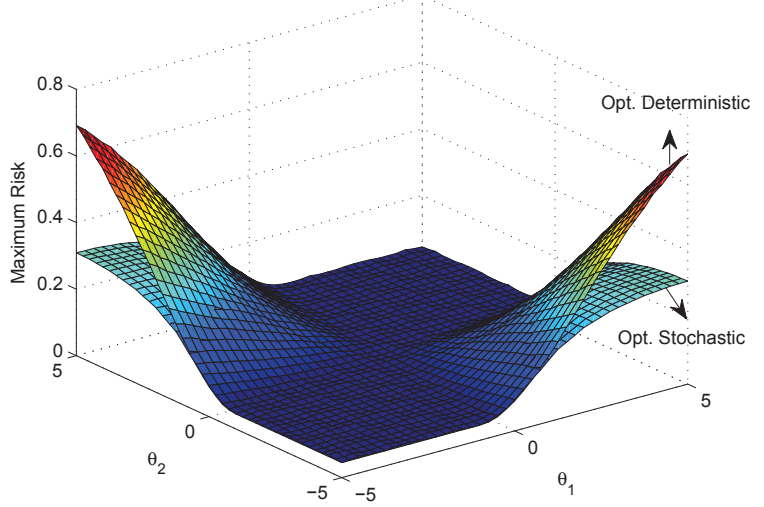

Fig. 5. Maximum Bayes risk versus $\boldsymbol{\theta}_{1}$ and $\boldsymbol{\theta}_{2}$.

proaches are presented for various values of $\boldsymbol{\theta}$. It can be observed that the maximum number of mass points is two for the total Bayes risk criterion, and it is three for the maximum Bayes risk criterion in accordance with the theoretical results.

\section{CONCLUSIONS}

In this study, the optimal stochastic design of multiple parameters has been investigated for a given set of fixed estimators. Two different performance criteria have been proposed; namely, the total Bayes risk criterion and the maximum Bayes risk criterion. It has been obtained that, in the presence of $K$ parameters, the optimal stochastic parameter design results in a randomization among at most two and $(K+1)$ different parameter values for the total and maximum Bayes risk criteria, respectively. The numerical examples have been presented to investigate the theoretical results, and to illustrate the level of improvements achievable via the proposed approach. 


\section{REFERENCES}

[1] S. M. Kay, Fundamentals of Statistical Signal Processing: Estimation Theory, Prentice Hall, Inc., Upper Saddle River, NJ, 1993.

[2] H. V. Poor, An Introduction to Signal Detection and Estimation, Springer-Verlag, New York, 1994.

[3] H. Soganci, S. Gezici, and O. Arikan, "Optimal stochastic parameter design for estimation problems," IEEE Trans. Sig. Processing, vol. 60, no. 9, pp. 4950-4956, September 2012.

[4] H. Chen, P. K. Varshney, S. M. Kay, and J. H. Michels, "Theory of the stochastic resonance effect in signal detection: Part I-Fixed detectors," IEEE Trans. Sig. Processing, vol. 55, no. 7, pp. 3172-3184, July 2007.

[5] S. M. Kay, "Noise enhanced detection as a special case of randomization," IEEE Sig. Processing Lett., vol. 15, pp. 709-712, 2008.

[6] S. Bayram, S. Gezici, and H. V. Poor, "Noise enhanced hypothesis-testing in the restricted Bayesian framework," IEEE Trans. Sig. Processing, vol. 58, no. 8, pp. 3972-3989, Aug. 2010.

[7] C. Goken, S. Gezici, and O. Arikan, "Optimal stochastic signaling for power-constrained binary communications systems," IEEE Trans. Wireless Commun., vol. 9, no. 12, pp. 3650-3661, Dec. 2010.

[8] B. Dulek and S. Gezici, "Detector randomization and stochastic signaling for minimum probability of error receivers," IEEE Trans. Commun., vol. 60, no. 4, pp. 923-928, Apr. 2012.

[9] H. Chen, P. K. Varshney, and J. H. Michels, "Noise enhanced parameter estimation," IEEE Trans. Sig. Processing, vol. 56, no. 10, pp. 5074-5081, Oct. 2008.

[10] A. Patel and B. Kosko, "Optimal noise benefits in Neyman-Pearson and inequality-constrained signal detection," IEEE Trans. Sig. Processing, vol. 57, no. 5, pp. 1655-1669, May 2009.

[11] S. Bayram and S. Gezici, "Stochastic resonance in binary composite hypothesis-testing problems in the Neyman-Pearson framework," Digital Signal Processing, vol. 22, no. 3, pp. 391-406, May 2012.

[12] D. P. Bertsekas, A. Nedic, and A. E. Ozdaglar, Convex Analysis and Optimization, Athena Specific, Boston, MA, 2003.

[13] K. E. Parsopoulos and M. N. Vrahatis, Particle swarm optimization method for constrained optimization problems, pp. 214-220, IOS Press, 2002, in Intelligent Technologies-Theory and Applications: New Trends in Intelligent Technologies.

[14] K. V. Price, R. M. Storn, and J. A. Lampinen, Differential Evolution: A Practical Approach to Global Optimization, Springer, New York, 2005. 